\title{
Zinc Sulfide Tubes Reinforced with Carbon Nanofibers
}

\author{
N. N. Kolesnikov, D. N. Borisenko, E. B. Borisenko, A. V. Timonina, and V. V. Kveder \\ Institute of Solid State Physics, Russian Academy of Sciences, Laboratory of Physical-Chemical Basis of Crystallization, \\ Chernogolovka, 142432 Moscow, Russia
}

Correspondence should be addressed to E. B. Borisenko, borisenk@issp.ac.ru

Received 10 October 2008; Revised 16 January 2009; Accepted 27 January 2009

Recommended by Burtrand Lee

Zinc sulfide submicron and nanotubes with outer diameters in the range from 100 to $1000 \mathrm{~nm}$ were produced through chemical deposition from vapor under argon pressure. The novel process provides formation of ZnS tubes reinforced with carbon nanofibers. This is the first time that the $\mathrm{ZnS}$ tubes are grown with fibers during deposition.

Copyright $\odot 2009$ N. N. Kolesnikov et al. This is an open access article distributed under the Creative Commons Attribution License, which permits unrestricted use, distribution, and reproduction in any medium, provided the original work is properly cited.

\section{Introduction}

Interest in nanocomposites increases due to their special properties and potential applications. Semiconductor II-VI composites are interesting because of their size-dependent optical properties, size-dependent band gap energies [1-3]. Core/shell products, for example, $\mathrm{ZnS} / \mathrm{HgS}, \mathrm{CdSe} / \mathrm{ZnS}$ fabricated by electro-deposition, or chemical solution transport $[1,2]$ are of special interest due to varying electronic properties dependent on core/shell weight ratio. In [3] $\mathrm{ZnS} / \mathrm{Si}$ core/shell tubes were fabricated by epitaxial growth. $\mathrm{ZnS}$ was a substrate used to produce Si tubes for semiconductor industry. ZnS templates were afterwards chemically removed. The core/shell structures are similar to the structures we obtain, but not the same.

We have developed a method of fabrication of $\mathrm{ZnS} / \mathrm{C}$ nanocomposite tubes by direct $\mathrm{ZnS}$ evaporation in a graphite crucible. This technique is a kind of CVD method: carbon from graphite crucible reacts with $\mathrm{ZnS}$. Then at lower temperature carbon deposits along with $\mathrm{ZnS}$ according to (1), so that the $\mathrm{ZnS}$ forms tubes and the tubes' walls are reinforced with the graphite nanofibers. This material is new and may be interesting as a reinforced semiconductor nanomaterial.

\section{Experimental}

Zinc sulfide tubes of the outer diameter 100-500 nm were grown in a graphite crucible of $40 \mathrm{~mm}$ in diameter and
$100 \mathrm{~mm}$ in height. The crucible was loaded with $100 \mathrm{~g}$ of zinc sulfide. The crucible was placed into the autoclave which had a graphite resistance heater. The setup is shown in Figure 1.

First, the pressure in the autoclave reached 1.3 . $10^{-2}$ mbar, then the chamber was filled with argon. The $\mathrm{ZnS}$ powder was preliminary annealed for $1 \mathrm{~h}$ at the temperature $600^{\circ} \mathrm{C}$ in argon atmosphere under pressure $52 \mathrm{~atm}$. After cooling to room temperature, the furnace was three times cleaned with high-purity argon and then heated to $1800^{\circ} \mathrm{C}$. The synthesis was performed for $1.5 \mathrm{~h}$ by the first reaction in (1) in the temperature range $1800-1850^{\circ} \mathrm{C}$ under $58 \mathrm{~atm}$ argon pressure, then the furnace was cooled and $\mathrm{ZnS}$ submicro and nanotubes deposited simultaneously with graphite nanofibers by the second reaction in (1):

$$
\mathrm{C}_{(S)}+\mathrm{ZnS}_{(V)} \stackrel{\geq 1800^{\circ} \mathrm{C}}{\longrightarrow} \mathrm{Zn}_{(V)}+\mathrm{CS}_{2} \stackrel{T \downarrow}{\longrightarrow} \mathrm{ZnS}_{\lfloor\text {nano }}+\mathrm{C}_{G N V}
$$

where $\mathrm{C}_{(S)}$ is graphite, $\mathrm{ZnS}_{(V)}$ is gaseous $\mathrm{ZnS}, \mathrm{C}_{G N V}$ is graphite nanofibers.

The $\mathrm{ZnS}$ submicro and nanotubes with carbon fibers were deposited on cool parts of the furnace (no. 7 in Figure 1). Macroscopically, the product of the reaction looked like gray soot. The deposited tubes were studied by transmission electron microscopy (TEM) on a JEM 100 CX TEM microscope. X-ray diffraction (XRD) patterns were taken on a Siemens 500D X-ray diffractometer, whereas electron probe microanalysis (EPMA) was performed on a VegaTescan setup. 


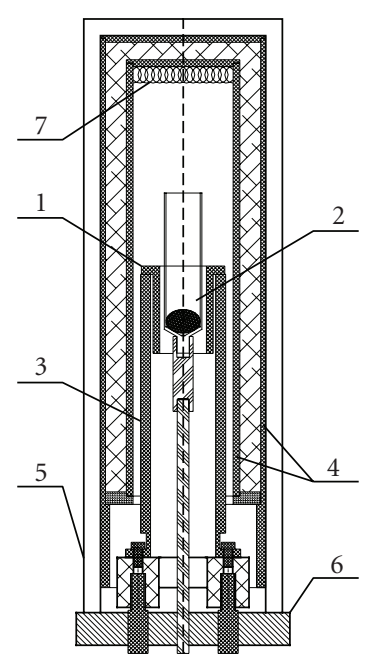

Figure 1: Scheme of the setup for growth of $\mathrm{ZnS}+\mathrm{C}$ nanocomposite: (1) heater, (2) graphite crucible with $\mathrm{ZnS}$ in the working position, (3) current leads, (4) heat screen, (5) case of high-pressure vessel (with water cooling jacket), (6) flange, and (7) soot with $\mathrm{ZnS}$ and graphite nanocomposite.

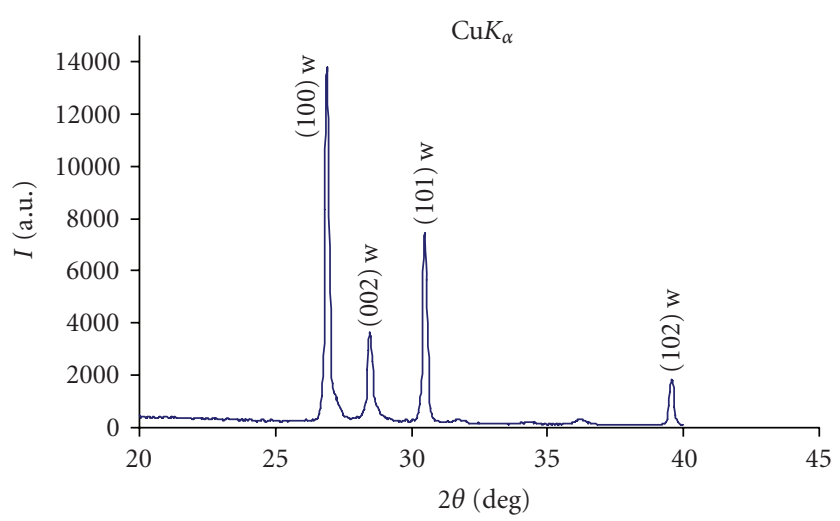

FIgURE 2: X-ray diffraction pattern of the deposited ZnS tubes.

The tubes were then etched in hot $\mathrm{HCl}$ and rinsed in distillated water. Some of these samples were annealed after etching at $870-890^{\circ} \mathrm{C}$ during 7 days, then cooled to room temperature. Microstructure, phase content and composition of the etched, and annealed samples were also studied by the abovementioned TEM, XRD, and EPMA techniques.

\section{Results and Discussion}

The X-ray diffraction pattern of the product of the reaction is shown in Figure 2. The product consists mostly of wurtzite $\mathrm{ZnS}$. Some carbon is sometimes present in the diffractograms.

Figure 3 shows (a) ZnS tubes, (b) the tube wall (3) containing channels (2) filled with graphite fibers (1), (c) the schematic view of a $\mathrm{ZnS}$ tube (3) containing channels (2)
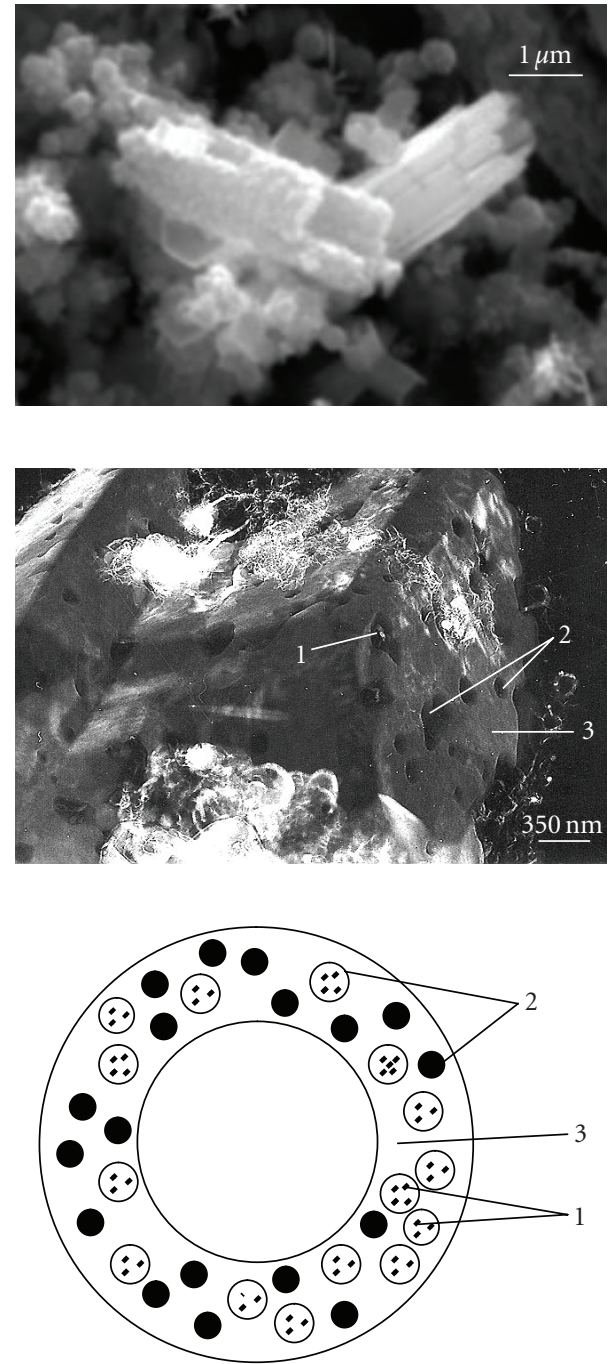

1- C nanotubes

2- Channels

3- ZnS tube

FIgURE 3: ZnS tubes deposited from vapor: (a) ZnS tubes; (b) ZnS tube walls with channels containing graphite nanofibers; (c) schematic diagram of $\mathrm{ZnS}$ tubes reinforced with $\mathrm{C}$ nanofibers: (1) graphite fibers, (2) channels, (3) ZnS tubes.

filled with graphite fibers (1). TEM image and the electron diffraction pattern of the grown $\mathrm{ZnS}$ crystals (2) containing C fibers (1) are shown in Figure 4 on the left. The electron diffraction pattern in the inset contains reflections from $\mathrm{ZnS}$ crystal and rings from carbon. The electron diffraction patterns were taken so that the incident beam was normal to the amorphous substrate. The $\mathrm{ZnS}$ tubes are randomly oriented on the surface. However, one zone $\langle 0001\rangle$ can be distinguished.

In the stable state $\mathrm{ZnS}$ has a cubic lattice with sphalerite structure. However, it undergoes a polymorphic transition at $1020^{\circ} \mathrm{C}$ when sphalerite transforms in wutrzite. In our case $\mathrm{ZnS}$ is quenched from the high-temperature state (see the reaction) and exists in hexagonal phase at room temperature. 

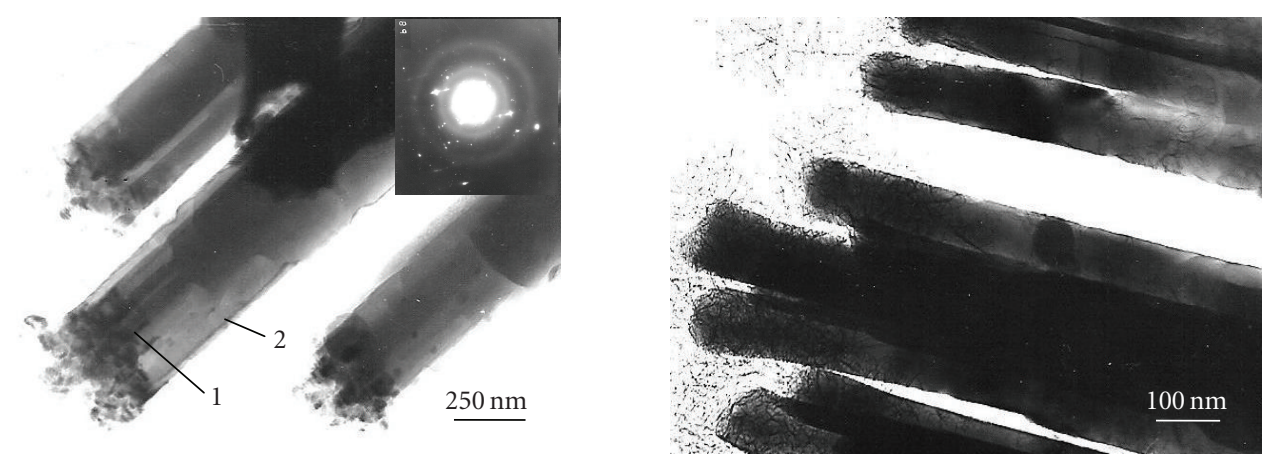

FIgURE 4: (1) ZnS tubes; on the right: graphite nanofibers, (2) ZnS tube; on the left: after etching in $\mathrm{HCl}$ a network of $\mathrm{C}$ fibers is revealed.
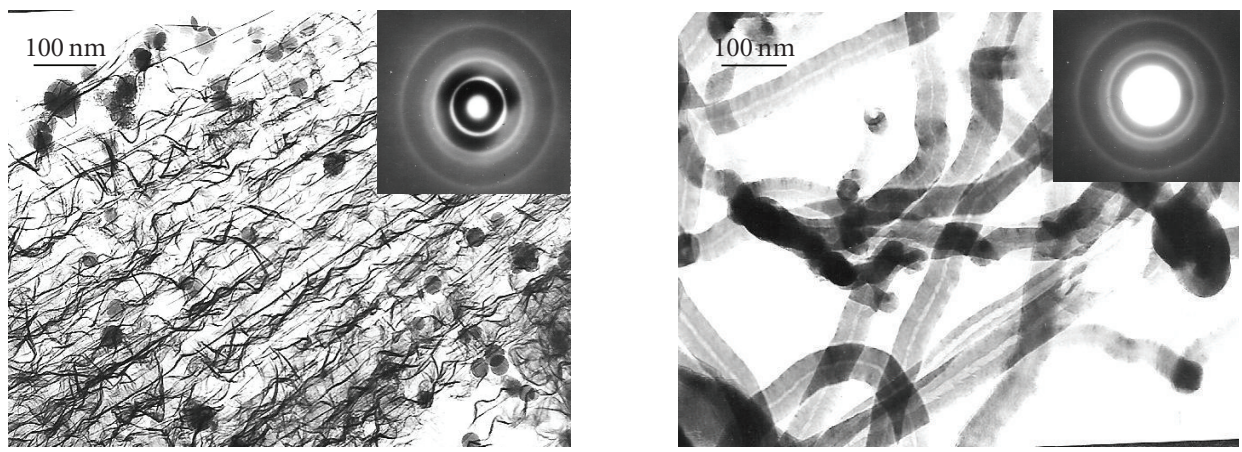

Figure 5: Graphite fibers from ZnS tubes remained after etching and annealing (on the left), annealing (on the right).

Intensities of the diffraction lines in the XRD patterns (Figure 2) differ from the ratio of intensities of a standard sample (JCPDS card 36-1450). These TEM diffraction and XRD data confirm that some grown directions prevail in the tubes, in particular, $c$ direction of the wurtzite structure.

The image in Figure 4 on the right is taken from the samples etched in hydrochloric acid. It is seen that etching in $\mathrm{HCl}$ causes dissolution of zinc sulfide and reveals network of carbon fibers.

The following annealing $\mathrm{ZnS}$ removes completely and only carbon fibers remain. This is seen from Figure 5 which shows the TEM images of the etched and annealed samples (left) and the annealed sample (right). In the insets on the left and on the right, electron diffraction patterns show rings from carbon.

It should be noted that the effect of annealing can be detected visually: the initially gray powder of $\mathrm{ZnS}$ and carbon mixture becomes absolutely black after annealing, which confirms indirectly that only carbon remains after quite long heating. White pure $\mathrm{ZnS}$ crystallites deposit on the cold end of the sealed tube. Semiquantitative EPMA microanalysis performed by energy-dispersion (ED) detection system confirms that only about $4 \%$ of $\mathrm{ZnS}$ remain after annealing, while $2 \%$ remain after combination of etching in hot $\mathrm{HCl}$ and the following annealing.

The experimental data show that during growth of $\mathrm{ZnS}$ submicro and nanotubes in graphite crucible the nanocomposite forms $\mathrm{ZnS}$ tubes reinforced with graphite nanofibers deposit as the furnace temperature is decreased.
The annealing or etching of the tubes removes $\mathrm{ZnS}$, so that only graphite nanofibers from the tube walls remain.

Thus, we have developed a chemical vapor deposition technique to produce $\mathrm{ZnS}$ submicro and nanotubes reinforced with graphite nanofibers.

\section{References}

[1] M. Kawa, H. Morii, A. Ioku, S. Saita, and K. Okuyama, "Largescale production of CdSe nanocrystal by a continuous flow reactor," Journal of Nanoparticle Research, vol. 5, no. 1-2, pp. 81-85, 2003.

[2] I. Chakraborty, D. Mitra, and S. P. Moulik, "Spectroscopic studies on nanodispersions of CdS, HgS, their core-shells and composites prepared in micellar medium," Journal of Nanoparticle Research, vol. 7, no. 2-3, pp. 227-236, 2005.

[3] J. Hu, Y. Bando, Z. Liu, J. Zhan, D. Goldberg, and T. Sekiguchi, "Synthesis of crystalline silicon tubular nanostructures with $\mathrm{ZnS}$ nanowires as removable templates," Angewandte Chemie International Edition, vol. 43, no. 1, pp. 63-66, 2003. 

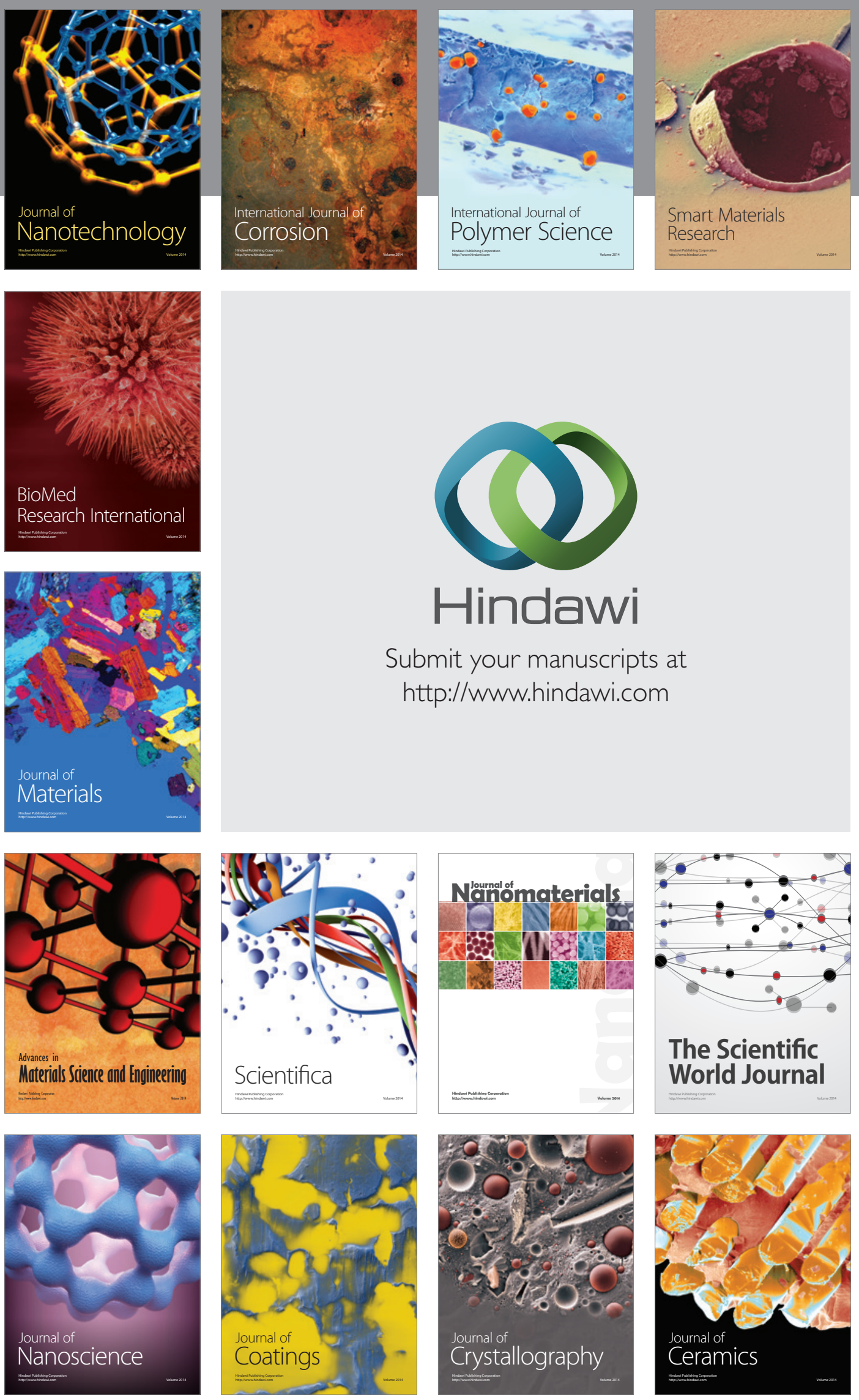

The Scientific World Journal

Submit your manuscripts at

http://www.hindawi.com

\section{World Journal}

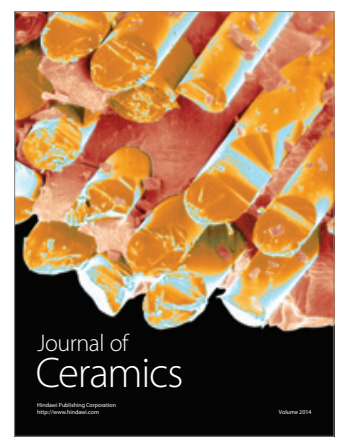

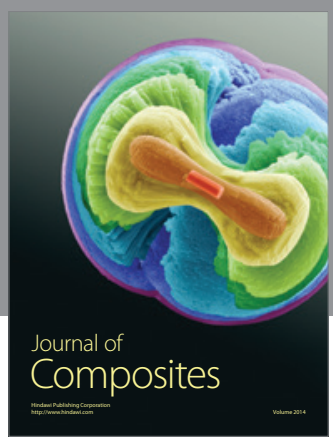
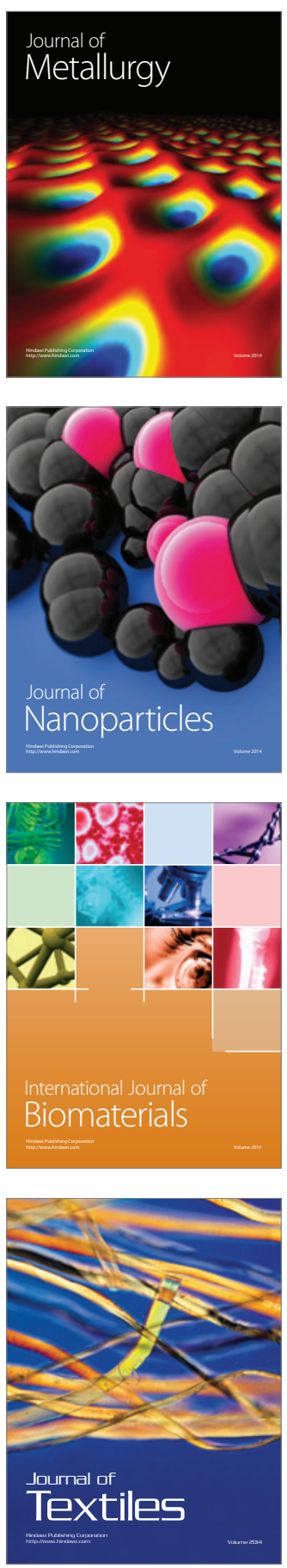